\title{
Optical Helmholtz resonators
}

\author{
Patrick Bouchon $^{1}$, Paul Chevalier ${ }^{1}$, and Riad Hadar ${ }^{1,2}$ \\ ${ }^{1}$ DOTA, ONERA, Universit Paris-Saclay, F-91123 Palaiseau, France \\ ${ }^{2}$ DOTA, ONERA, Universit Paris-Saclay, F-91123 Palaiseau, France \\ *corresponding author, E-mail: patrick. bouchon@onera. fr
}

\begin{abstract}
Optical nanoantennas are studied to manipulate light and enhance light matter interactions. Here, we experimentally demonstrate the optical Hemholtz resonance in a metallic slit-box structure, which is predicted to be harmonic and to enhance the electric field intensity by several orders of magnitude. It is fabricated thanks to a two steps electron beam lithography, between which the box was filled with benzocyclobutene (BCB).
\end{abstract}

\section{Introduction}

Designing nanoantenna that could strongly and efficiently concentrate incident light into deep subwavelength volumes is a key issue to locally enhance the electric field and thus produce strong light-matter interactions. Many existing designs are inspired by structures widely used in the radiofrequency domain such as bowtie or Yagi-Uda antennas. Here, we rather use an analogy between acoustics and electromagnetism wave equations, in order to adapt the acoustic Helmholtz resonator to optics. This resonator, made of a slit-box structure, behave as a LC circuit. The deep subwavelength slit acts as a capacitance, which concentrates the electric field at resonance, leading to huge enhancement in a hot volume. Here, we present a way to fabricate this nanoantenna, and experimentally demonstrate the optical Helmholtz resonance.

\section{2. the Optical Helmholtz Resonator}

\subsection{Properties}

This structure is made of a tiny slit above a larger cavity (see Fig. 1(a)), and exhibits several appealing features: total absorption at resonance, absence of harmonic resonance, giant field intensity enhancement in the whole slit volume, angular independence of the Helmholtz resonance $[1,3]$.

The key properties of the Helmholtz resonance are:

(i) A giant field enhancement, typically two orders of magnitude higher than expected with gap plasmons resonances.

(ii) A single resonance behavior with no harmonics.

(iii) No influence of the permittivity of the material filling the cavity on the resonance wavelength.

(iv) The resonance wavelength $\lambda_{r}$ depends on the square root of all four geometrical parameters according to the following formula:

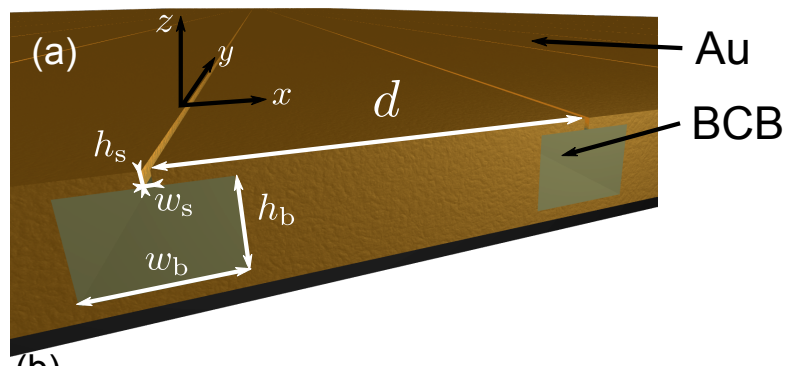

(b)

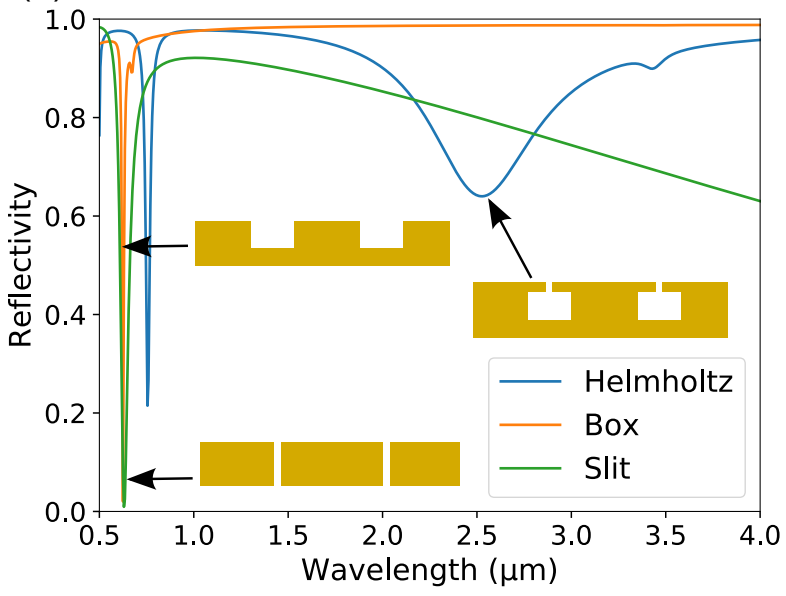

Figure 1: (a) General schematic of the optical Helmholtz resonator, made of a box and a slit in metal, both infinite along the $y$ direction. (b) Computed reflectivity spectrum of the Helmholtz resonator array (period $d=450 \mathrm{~nm}$ ) made of a slit (width $w_{s}=52 \mathrm{~nm}$, height $h_{s}=37 \mathrm{~nm}$ ) and a box (width $w_{b}=400 \mathrm{~nm}$, height $h_{b}=77 \mathrm{~nm}$ ), compared with the reflectivity spectra of the array of slits (height $4 h_{s}$, width $w_{s}$, orange curve) and boxes (height $h_{b}$, width $w_{b}$,green curve). The impinging light is normally incident and TM-polarized.

$$
\lambda_{r} \propto 2 \pi \sqrt{\varepsilon_{s} \frac{h_{b} w_{b} h_{s}}{w_{s}}},
$$

where $\varepsilon_{s}$ is the permittivity of the material filling the slit.

\subsection{Comparison to other resonances}

The computed absorption spectrum, for a transverse magnetic (TM) polarized and normally incident light, is plot- 
(a)

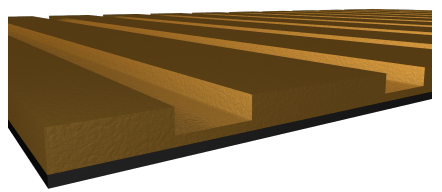

(b)

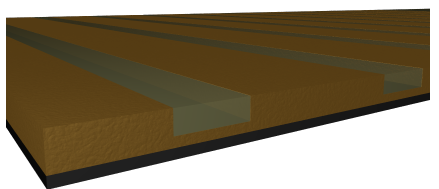

(c)

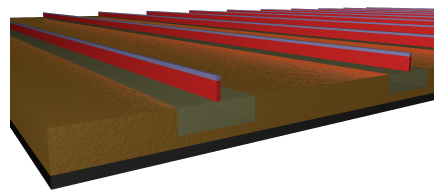

(d)
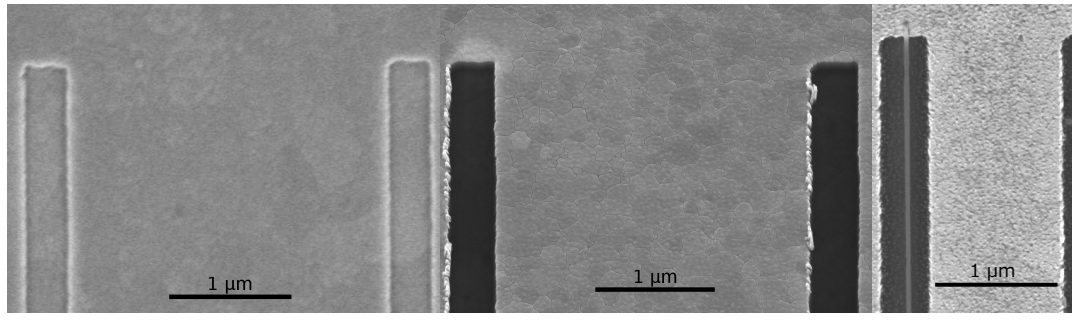

$1 \mu \mathrm{m}$
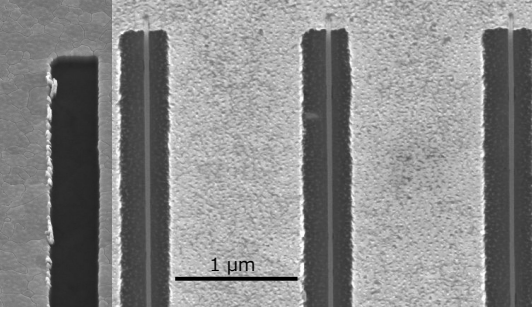
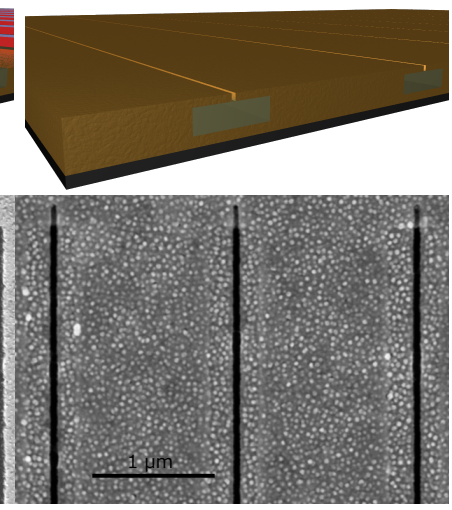

Figure 2: Fabrication steps for a periodic grating of optical Helmholtz resonators with the corresponding top view SEM image: (a) Gold trenches are created by the lift-off of $80 \mathrm{~nm}$ of gold after a first e-beam lithography $(d=3 \mu \mathrm{m})$.(b) A dielectric (BCB) is spin coated on the surface and etched by RIE to reach the gold surface $(d=3 \mu \mathrm{m})$. (c) A two layer lithography process is performed using PMMA and HSQ $(d=1.5 \mu \mathrm{m})$. (d) Gold is lifted-off resulting in the fabrication of the optical Helmholtz resonator $(d=1.5 \mu \mathrm{m})$. The narrow slits in the gold surface above the BCB-filled cavity, which is barely distinguishable.

ted in Fig. 1(b) for a given geometry $\left(w_{\mathrm{b}}=400 \mathrm{~nm}\right.$, $h_{\mathrm{b}}=77 \mathrm{~nm}, w_{\mathrm{s}}=52 \mathrm{~nm}, h_{\mathrm{s}}=37 \mathrm{~nm}, d=450 \mathrm{~nm}$ ). At $\lambda=2.53 \mu \mathrm{m}, 36 \%$ of the light is absorbed due to the Helmholtz resonance. Other resonances appear at lower wavelengths between $500 \mathrm{~nm}$ and $700 \mathrm{~nm}$. The small peak at $\lambda=3.4 \mu \mathrm{m}$ is due to a vibrational absorption of BCB that is enhanced by the Helmholtz resonator. For the sake of comparison, the absorption spectra are also plotted under the same illumination conditions for an array of slits (height $4 h_{\mathrm{s}}$, width $w_{\mathrm{s}}$ ) and for an array of cavity filled with BCB (width $w_{\mathrm{b}}$, height $h_{\mathrm{b}}$ ) that also exhibit resonant absorption, but at lower wavelengths.

\subsection{Fabrication}

Due to its complex architecture and its deeply subwavelength dimensions, the fabrication of the Helmholtz resonator is a rather complex challenge. We have developed a process in two parts, the first consists in fabricating the cavity, which is subsequently filled with benzocyclobutene (BCB), a planarizing dielectric, as shown in Fig. 1(a) to reduce the complexity of fabrication by avoiding freestanding parts. A first step of e-beam lithography is performed to define the cavity on top of the gold layer. $80 \mathrm{~nm}$ of gold are evaporated onto the sample and lifted-off (see Fig. 2(a)) to fabricate trenches of height $80 \mathrm{~nm}$ and width $400 \mathrm{~nm}$. Benzocyclobutene (BCB) is spin coated on the sample and baked, then a reactive ion etching (RIE) plasma is done so that the surface of the $\mathrm{BCB}$ reaches the gold surface while keeping the cavity filled with dielectric (see Fig. 2(b)). On top of theses trenches, a bi-layer resist stack (PMMA-HSQ) is used to define the slit with a fine resolution. The second level is aligned on the lower level with alignment markers positioned during the first step of lithography and detected by the e-beam pattern generator. The accuracy of this alignment on the sample size is about $50 \mathrm{~nm}$, and a slight shift of the slit in regard to the cavity can be seen on the SEM im- ages (see Fig. 2(c-d)). Near and mid infrared with a Fourier transform infrared spectrometer confirms the presence of the Helmholtz resonance at $\lambda_{r}=2.84 \mu \mathrm{m}$.

\section{Conclusion}

A process involving a two-steps e-beam lithography was used to fabricate optical Helmholtz resonators. It was used to experimentally demonstrate the optical Helmholtz resonance thanks to measurements in the near and mid-infrared. This resonators produces high field intensity enhancement that can be used to enhance SEIRA.

\section{Acknowledgement}

We acknowledge financial support from the ONERA through the MOLIERE project and from the Agence Nationale de la Recherche (ANR) (ANR-16-CE39-0007)

\section{References}

[1] P. Chevalier, P. Bouchon, R. Hadar, and F. Pardo, Optical Helmholtz resonators, Appl. Phys. Lett. 105: 071110, 2014

[2] P. Chevalier, P. Bouchon, E. Sakat, J.L. Pelouard, F. Pardo, and R. Hadar, Extraordinary transmission in optical Helmholtz resonators, Opt. Lett. 40: 2735, 2015

[3] P. Chevalier, P. Bouchon, J.J Greffet, J.L. Pelouard, R. Hadar, and F. Pardo, Giant field enhancement in electromagnetic Helmholtz nanoantenna, Phys. Rev. B 90: 195412,2014

[4] P. Chevalier, P. Bouchon, et al., Experimental demonstration of the optical Helmholtz resonance, Appl. Phys. Lett. 112, 171110 (2018) 